\title{
Muon front end for the neutrino factory
}

\author{
C. T. Rogers* \\ STFC Rutherford Appleton Laboratory, Harwell Science and Innovation Campus, Didcot, OX11 OQX, United Kingdom \\ D. Stratakis \\ Brookhaven National Laboratory, Upton, New York 11973-5000, USA
}

G. Prior and S. Gilardoni

European Organization for Nuclear Research CERN, CH-1211 Genève 23, Switzerland

D. Neuffer

Fermi National Laboratory, P.O. Box 500, Batavia, Illinois 60510-5011, USA

P. Snopok

Illinois Institute of Technology, 3300 South Federal Street, Chicago, Illinois 60647, USA

A. Alekou

Imperial College London, Blackett Laboratory, Prince Consort Road, London SW7 2BW, United Kingdom

J. Pasternak

Imperial College London, Blackett Laboratory, Prince Consort Road, London, SW7 2BW, United Kingdom, and STFC Rutherford Appleton Laboratory, Harwell Science and Innovation Campus, Didcot, OX11 0QX, United Kingdom

(Received 9 November 2012; published 24 April 2013)

\begin{abstract}
In the neutrino factory, muons are produced by firing high-energy protons onto a target to produce pions. The pions decay to muons and pass through a capture channel known as the muon front end, before acceleration to $12.6 \mathrm{GeV}$. The muon front end comprises a variable frequency rf system for longitudinal capture and an ionization cooling channel. In this paper we detail recent improvements in the design of the muon front end.
\end{abstract}

DOI: 10.1103/PhysRevSTAB.16.040104

PACS numbers: 29.20.Ej, 14.60.Pq

\section{INTRODUCTION}

In the neutrino factory [1], a $4 \mathrm{MW}$ proton beam is delivered onto a target to create a flux of pions, muons, and background particles. The proton beam is delivered in a train of 1-3 bunches produced at $50 \mathrm{~Hz}$. In order for $\mathrm{rf}$ cavities in the muon acceleration chain to refill, proton bunches are produced with at least $50 \mu$ s intervals.

The neutrino factory muon front end captures secondary particles produced on the target by means of a pion decay channel and particle selection system followed by a longitudinal drift to an adiabatic buncher, energy-phase rotation system, and ionization cooling channel, after which the beam enters a chain of muon accelerators.

Pions are captured in a solenoidal field that tapers adiabatically from 20 to $1.5 \mathrm{~T}$ while the beam pipe radius

\footnotetext{
*chris.rogers@stfc.ac.uk
}

Published by the American Physical Society under the terms of the Creative Commons Attribution 3.0 License. Further distribution of this work must maintain attribution to the author(s) and the published article's title, journal citation, and DOI. increases from 0.075 to $0.3 \mathrm{~m}$. A large transverse phase space of both positively and negatively charged particles with a large energy spread is captured in the solenoidal field. Protons and other background particles are removed from the beam by means of a bent-solenoid chicane and proton absorber and subsequently a time-energy relationship is allowed to develop.

The beam passes through a series of rf cavities, each of which has frequency selected to be synchronous with particles in the beam and the voltage of each one higher than the previous to adiabatically form microbunches. Once the voltage has reached its nominal peak value, the cavity frequency is chosen so that fast muons at the head of the bunch experience a decelerating phase and slow muons at the tail of the bunch experience an accelerating phase. The phase is chosen so that the head bunch and tail bunch have the same energy when the bunch frequency is $201.25 \mathrm{MHz}$.

Subsequently, the beam enters an ionization cooling channel. The particles are passed through material absorbers reducing both transverse and longitudinal momentum. They are then reaccelerated in $\mathrm{rf}$ cavities replacing momentum in only the longitudinal direction resulting in 
a reduction of transverse emittance. Multiple Coulomb scattering and energy straggling tend to create noise counteracting the emittance reduction. A low- $Z$ material (lithium hydride) is chosen for the absorber to minimize these effects.

In order to maintain a high acceptance, solenoids are tightly packed around rf cavities and absorbers. There are indications that this can facilitate breakdown in the cavities, which may make the baseline parameters challenging to achieve.

Following the muon front end, particles are passed into an acceleration system, accelerated to $12.6 \mathrm{GeV}$, and placed in a storage ring where the majority decay radioactively to form neutrinos. In the baseline, at least $10^{21}$ muon decays in the production straight are required per year.

The present design is based on the lattice presented in the neutrino factory study $2 \mathrm{~A}$ report [2] and subsequently developed in the International Scoping Study for a Neutrino Factory (ISS) [3] with several modifications: a particle selection system has been introduced to manage secondaries from the target; the solenoid-field strength in the drift, buncher, and phase rotation sections has been reduced from 1.75 to $1.5 \mathrm{~T}$; the whole system has been shortened; and the thickness of the lithium hydride absorbers in the cooling section has been increased. Additionally, a significant effort has been invested in designing alternate schemes for ionization cooling to mitigate the effect of $\mathrm{rf}$ breakdown that may be caused by overlapping magnetic fields with the cavities.

In this paper we summarize these improvements.

\section{DECAY REGION AND PARTICLE SELECTION SCHEME}

In this study, the input beam to the neutrino factory was taken to be composed of a monochromatic $8 \mathrm{GeV}$ proton beam with a 3 ns time spread incident on a liquid mercury target in the bore of a $20 \mathrm{~T}$ solenoid. The interaction of the beam with the target was modeled using the MARS15 (2010) default generator [4].

After the target, the field tapers from 20 to $1.5 \mathrm{~T}$ over a distance of $15 \mathrm{~m}$ collecting both positive and negative particle species. A large flux of protons of all energies comes off the target up to the energy of incoming protons. Additionally, relatively low-energy electrons and pions are produced. The particles pass through a solenoidal chicane where they acquire a vertical dispersion, with oppositely charged particles gaining a dispersion in the opposite direction. Subsequently, particles pass through a beryllium absorber, which removes almost all of the remaining, lowenergy, protons.

\section{A. Chicane}

The chicane starts $30 \mathrm{~m}$ after the proton target. The radius of curvature of the chicane is $22.92 \mathrm{~m}$, with a $12.5^{\circ}$ outward bend followed by a $12.5^{\circ}$ return bend.
This results in a transverse displacement of the decay section by $1.09 \mathrm{~m}$. This prevents the highest energy particles, that are relatively unperturbed by the magnetic field, from passing straight through the chicane. It gives momentum collimation for momenta greater than $500 \mathrm{MeV} / c$, which is the upper limit for capture by the phase rotation system. By maintaining the symmetry of the chicane, particles within the momentum acceptance are returned to the axis with very little emittance growth.

A simulation of a shell of particles passing through the chicane with initial transverse amplitude of $50 \mathrm{~mm}$ is shown in Fig. 1. The emittance change of particles as a function of momentum is shown together with a projection of the $x, y$ position of the particles after the chicane for a variety of momenta. There is a clear transition at $500-550 \mathrm{MeV} / c$ where particles are no longer transported by the chicane. Below this transition particles are
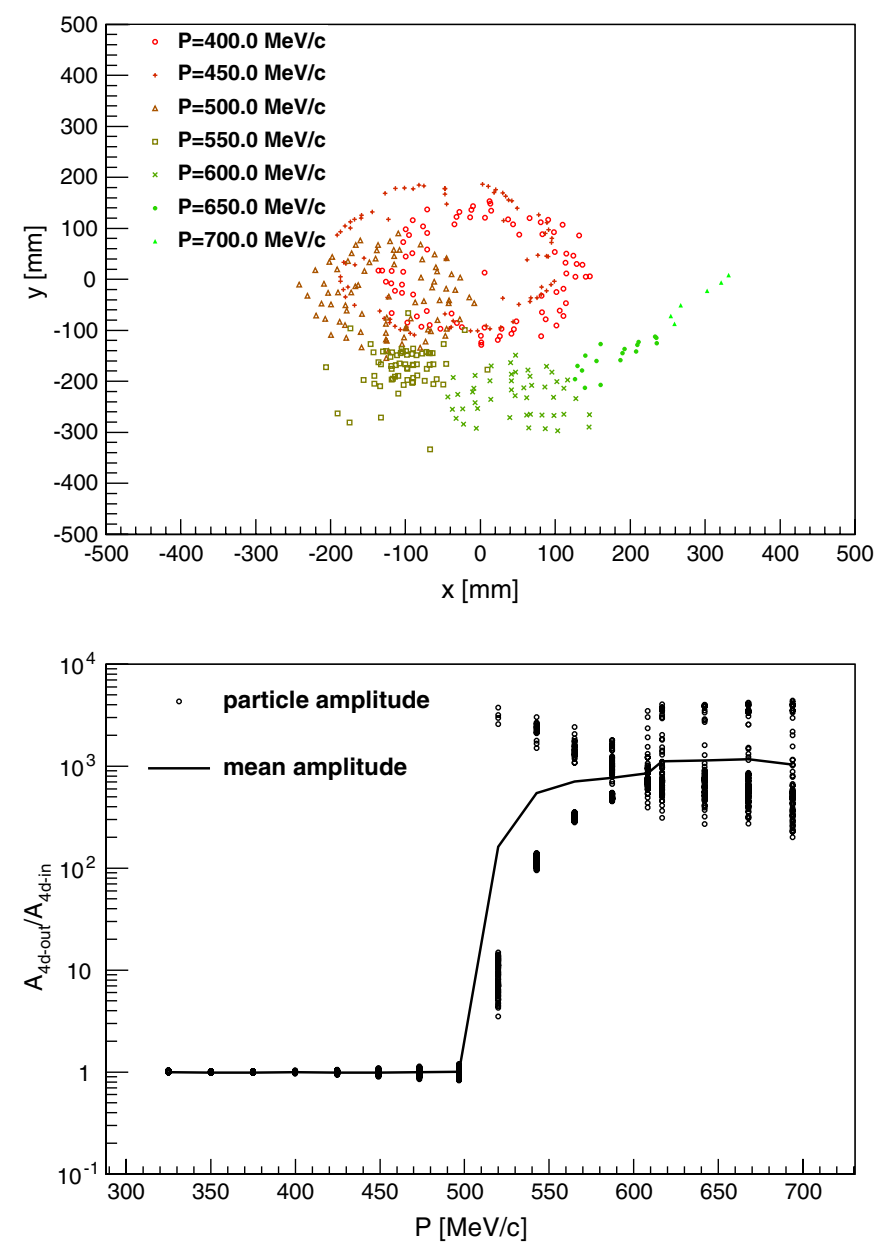

FIG. 1. (top) $x-y$ projection and (bottom) fractional change in transverse amplitude of a shell of muons after transport through the chicane system for a variety of momenta. The muons were created on the surface of a hyperellipsoid in $\left(x, p_{x}, y, p_{y}\right)$ space at intervals of $25 \mathrm{MeV} / c$ total momentum. The surface was chosen at each momentum to be constant in the $1.5 \mathrm{~T}$ field with 4D transverse amplitude of $50 \mathrm{~mm}$. Simulation in MAUS 0.3.1 [5]. 
transported with little or no emittance growth, even for a relatively high emittance beam with a very large momentum spread.

\section{B. Proton absorber}

Immediately following the chicane a beryllium absorber is placed in the beam line to remove the remaining proton impurities. Protons with energies in the sub-GeV regime are stopped in considerably less material than lighter particles of the same energy. Stochastic effects can lead to some protons receiving a smaller energy loss and passing through the absorber even for relatively high proton
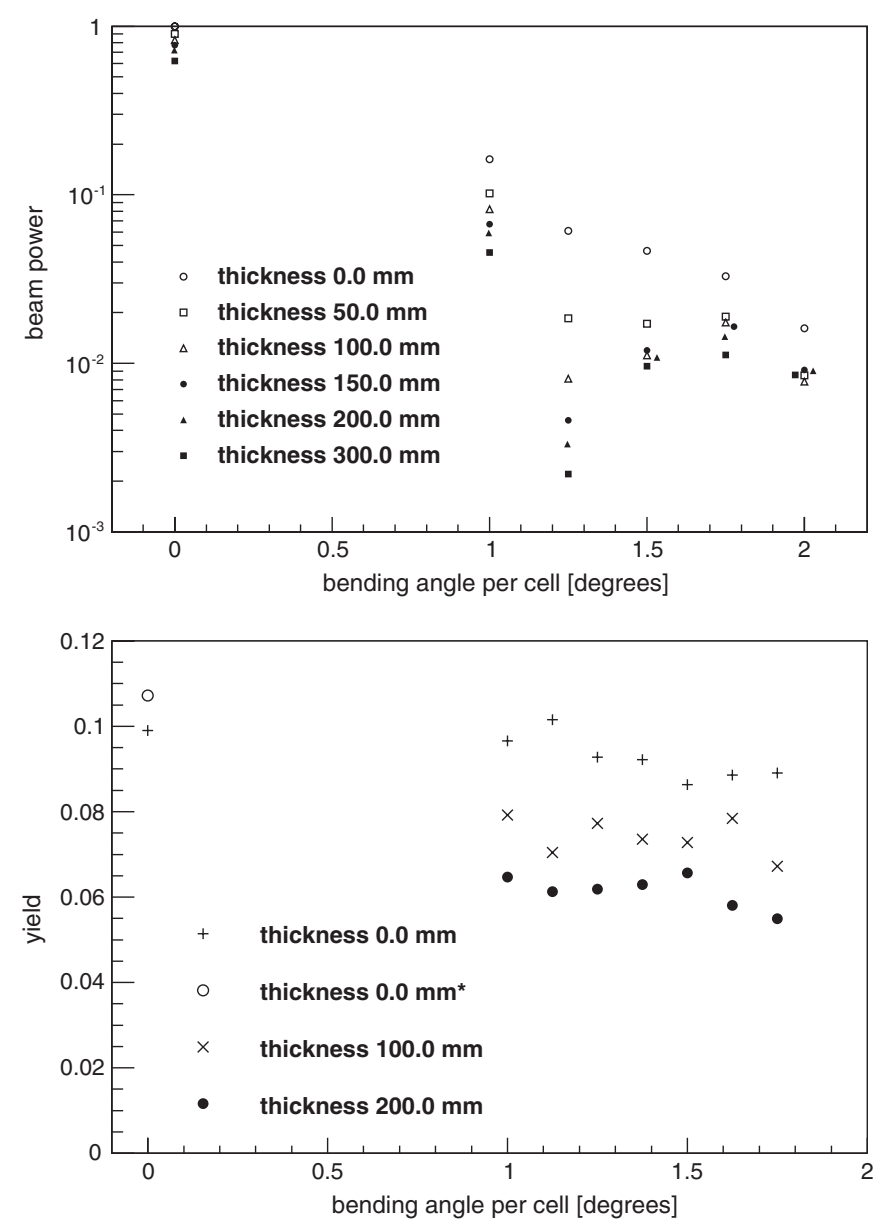

FIG. 2. (top) Fractional power of protons and (bottom) good muon yield at the end of the cooling channel for different chicane bending angles and absorber thicknesses. Overlapping points have been laterally displaced for clarity. The bending angle per cell is shown; each arc of the chicane was simulated with ten two-coil cells, each of which was rotated in the simulation to provide a smoothly varying solenoid field. No dipole field was applied. A cylindrical beryllium absorber was placed after the chicane with thicknesses listed. The two points for $0 \mathrm{~mm}$ thickness and $0^{\circ}$ bending angle in the bottom figure assume $(+)$ ideal constant $1.5 \mathrm{~T}$ field and (*) 3D magnetic field maps. All chicane simulations use realistic magnet field maps. Simulation in G4BEAMLINE 2.06 [6]. absorber thicknesses. The absorber can also create emittance growth in the muon beam. There is a tension between improving the muon yield with a thinner absorber and decreasing the proton yield with a thicker absorber.

In Fig. 2 the total proportion of protons and the yield of good muons per proton is shown as a function of chicane bending angle for various absorber thicknesses. There is a good optimum with a bending angle of $12.5^{\circ}\left(1.25^{\circ}\right.$ per cell $)$ and a proton absorber thickness of $100 \mathrm{~mm}$.

Roughly $0.8 \%$ of the proton beam power (of order $1 \mathrm{~kW}$ ) remains in the beam. These protons deposit most of their energy in the cooling channel. It is envisaged that a short remote handling section, possibly associated with a precooler, will be necessary around the start of the cooling section to handle these remaining protons.

Beryllium was chosen as a material for the proton absorber as it does not react with water coolants, it is robust to thermal stressing and has low atomic number. In general, low atomic number materials cause less scattering and associated beam heating.

\section{Loss handling}

Several $100 \mathrm{kWs}$ of beam power are deposited in the chicane and a significant amount of energy is deposited by showering electrons after the proton absorber. The chicane is comprised mainly of normal conducting coils in order to manage such losses. The electromagnetic shower after the proton absorber is produced mainly by relatively lowenergy electrons and photons and so direct shielding should be effective in the area downstream of the absorber.

\section{IMPROVEMENTS TO THE RF CAPTURE SYSTEM}

The rf capture system has been optimized to produce a shortened bunch train by reducing the length of the drift section and other regions. The baseline design delivers a bunch train that is less than $80 \mathrm{~m}$ long. This is an improvement over the version of the design developed for the ISS [3] which delivered a $120 \mathrm{~m}$ long bunch train containing the same number of muons. A shorter bunch train makes some downstream systems easier to design.

\section{A. Buncher}

To determine the required buncher parameters, we consider reference particles $\left(0, N_{B}\right)$ at $p_{0}=233 \mathrm{MeV} / c$ and $p_{N_{B}}=154 \mathrm{MeV} / c$, with the intent of capturing muons from an initial kinetic energy range of 50 to $400 \mathrm{MeV}$. The rf cavity frequency, $f_{\mathrm{rf}}$, and phase are set to place these reference particles at the center of bunches while the $\mathrm{rf}$ voltage increases along the channel. These conditions can be maintained if the rf wavelength, $\lambda_{\mathrm{rf}}$, increases along the buncher, following 
TABLE I. Summary of front-end rf requirements. Where power requirement is listed per frequency, this is the requirement per group of $\mathrm{rf}$ cavities of the same frequency. Where it is listed per cavity, this is the requirement per single rf cavity. The total installed rf voltage is $1184 \mathrm{MV}$.

\begin{tabular}{lcccccc}
\hline \hline & $\begin{array}{c}\text { Length } \\
{[\mathrm{m}]}\end{array}$ & $\begin{array}{c}\text { Number of } \\
\text { cavities }\end{array}$ & $\begin{array}{c}\text { Frequencies } \\
{[\mathrm{MHz}]}\end{array}$ & $\begin{array}{c}\text { Number of } \\
\text { frequencies }\end{array}$ & $\begin{array}{c}\text { Peak gradient } \\
{[\mathrm{MV} / \mathrm{m}]}\end{array}$ & $\begin{array}{c}\text { Peak power } \\
\text { requirements }\end{array}$ \\
\hline Buncher & 33.0 & 33 & 319.6 to 233.6 & 13 & 3.42 to 9.01 & $1-3.5 \mathrm{MW} /$ frequency \\
Rotator & 42.0 & 56 & 230.2 to 202.3 & 15 & 13 & $2.5 \mathrm{MW} /$ cavity \\
Cooler & 97.5 & 130 & 201.25 & 1 & $4 \mathrm{MW} / \mathrm{cavity}$ \\
Total & 172.5 & 219 & 319.6 to 201.25 & 29 & $\mathrm{MW}$ \\
\hline \hline
\end{tabular}

$$
N_{B} \lambda_{\mathrm{rf}}(s)=N_{B} \frac{c}{f_{\mathrm{rf}}(s)}=s\left(\frac{1}{\beta_{N_{B}}}-\frac{1}{\beta_{0}}\right)
$$

where $s$ is the total distance from the target, $\beta_{0}$ and $\beta_{N_{B}}$ are the velocities of the reference particles, and $N_{B}$ is an integer. For the present design, $N_{B}$ is chosen to be 10 , and the buncher length is $33.0 \mathrm{~m}$. With these parameters, the rf cavities decrease in frequency from $320 \mathrm{MHz}\left(\lambda_{\mathrm{rf}}=\right.$ $0.94 \mathrm{~m})$ to $230 \mathrm{MHz}\left(\lambda_{\mathrm{rf}}=1.3 \mathrm{~m}\right)$ over the length of the buncher.

The initial geometry for the placement of the $\mathrm{rf}$ cavities uses $0.4-0.5 \mathrm{~m}$ long cavities placed within $0.75 \mathrm{~m}$ long cells. The $1.5 \mathrm{~T}$ solenoid focusing of the decay region is continued through the buncher and the rotator section which follows. The rf gradient is increased from cell to cell along the buncher, and the beam is captured into a string of bunches, each of which is centered about a test particle position, with energies determined by the spacing from the initial test particle such that the $i$ th reference particle has velocity

$$
1 / \beta_{i}=1 / \beta_{0}+\frac{i}{N_{B}}\left(\frac{1}{\beta_{N_{B}}}-\frac{1}{\beta_{0}}\right) .
$$

In the initial design, the cavity gradients, $V_{\mathrm{rf}}$, follow a linear increase along the buncher:

$$
V_{\mathrm{rf}}(z) \approx 9 \frac{z}{L_{B}} \mathrm{MV} / \mathrm{m}
$$

where $z$ is distance along the buncher and $L_{B}$ is the length of the buncher. The gradient at the end of the buncher is $9 \mathrm{MV} / \mathrm{m}$. This gradual increase of the bunching voltage enables a somewhat adiabatic capture of the muons into separated bunches, which minimizes phase-space dilution.

In the practical implementation of the buncher concept, this linear ramp of cavity frequency is approximated by a sequence of rf cavities that decrease in frequency along the $33 \mathrm{~m}$ beam transport allotted to the buncher. The number of different $\mathrm{rf}$ frequencies is limited to a more manageable 13 (1-4 rf cavities per frequency). The linear ramp in gradient described by (3) is approximated by the placement and gradient of the cavities in the buncher. Table I shows a summary of the rf cavities that are needed in the buncher, rotator, and cooling sections.

\section{B. Rotator}

In the rotator section, the $\mathrm{rf}$ bunch spacing between the reference particles is shifted away from the integer, $N_{B}$, by an increment, $\delta N_{B}$, and phased so that the high-energy reference particle is stationary and the low-energy one is uniformly accelerated to arrive at the same energy as the first reference particle at the end of the rotator. For the baseline, $\delta N_{B}=0.05$ and the bunch spacing between the reference particles is $N_{B}+\delta N_{B}=10.05$. This is accomplished using an rf gradient of $13 \mathrm{MV} / \mathrm{m}$ in $0.5 \mathrm{~m}$ long rf cavities within $0.75 \mathrm{~m}$ long cells. The $\mathrm{rf}$ frequency decreases from 230.2 to $202.3 \mathrm{MHz}$ along the length of the $42 \mathrm{~m}$ long rotator region. A schematic of a rotator cell is shown in Fig. 3.
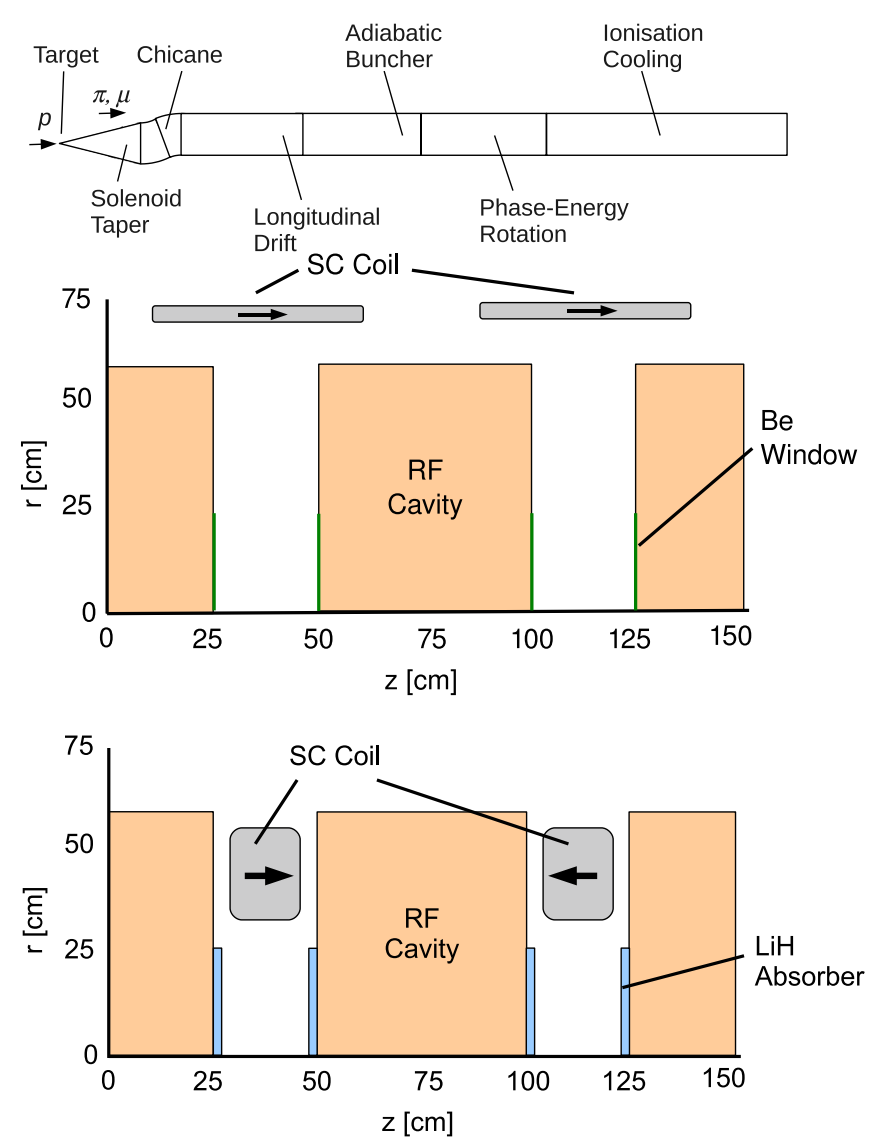

FIG. 3. (top) Schematic of the muon front end, (middle) rotator lattice, and (bottom) cooling lattice. 
The $\mathrm{rf}$ frequency is set by requiring that the trajectories of the reference particles be spaced in $c t$ by $\left(N_{B}+\delta N_{B}\right)$ wavelengths. In a practical implementation, a continuous change in frequency from cavity to cavity is replaced by grouping adjacent sets of cavities into the same rf frequency. The $42 \mathrm{~m}$ long rf rotator then contains $56 \mathrm{rf}$ cavities grouped into 15 frequencies.
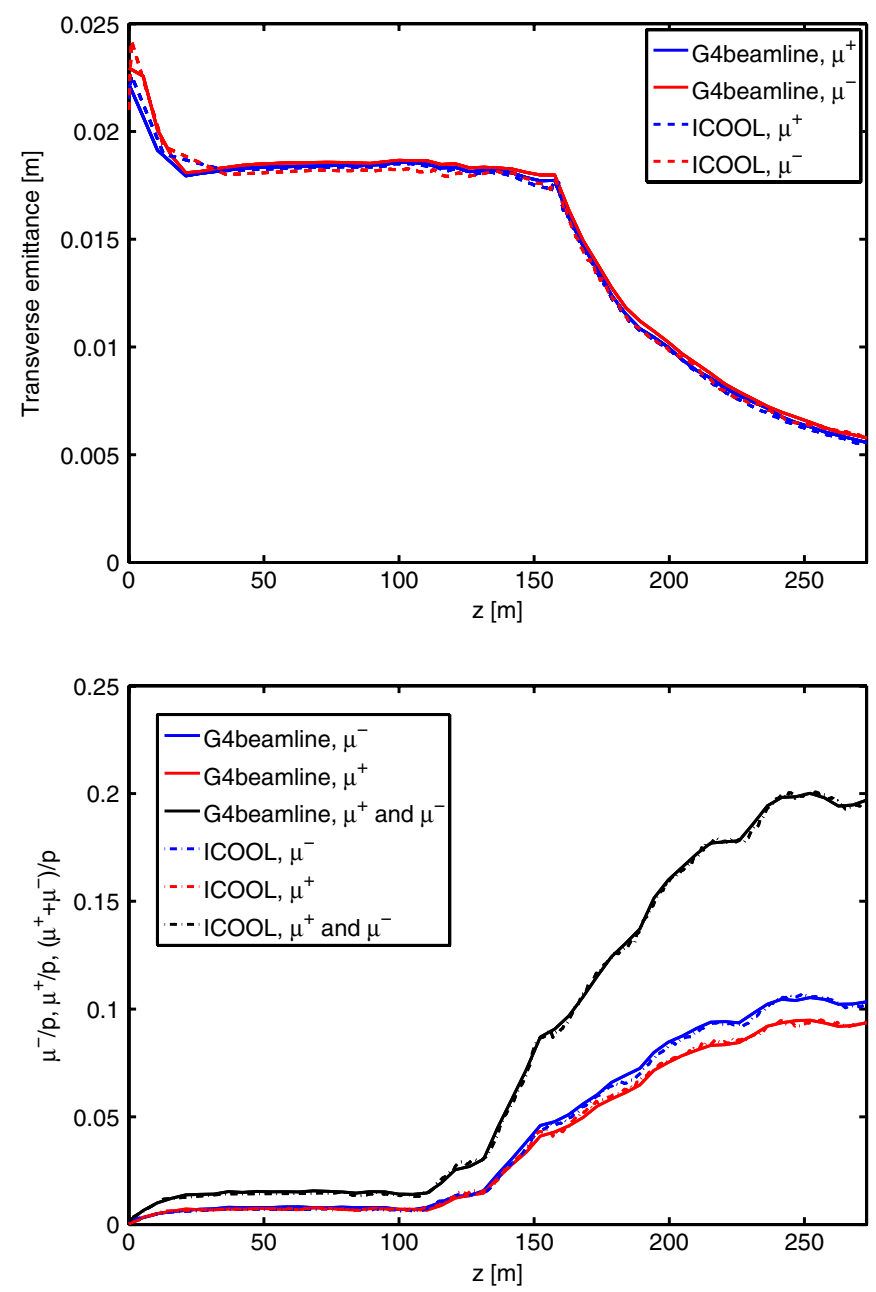

FIG. 4. Performance of the bunching and cooling channel as a function of distance along the channel, as simulated using the ICOOL 3.20 [7] and G4BEAMLINE 2.06 [6]. (top) The evolution of the rms transverse emittance (computed over all bunches). (bottom) The evolution of the number of muons within a reference acceptance (muons within $201.25 \mathrm{MHz}$ rf bunches with momentum in the range $100-300 \mathrm{MeV} / c$, transverse amplitude squared less than $0.03 \mathrm{~m}$ and longitudinal amplitude squared less than $0.15 \mathrm{~m}$ ). The cooling section starts at $s=$ $155 \mathrm{~m}$, where the rms transverse emittance is $0.018 \mathrm{~m}$ and $0.08 \mu$ per proton are in the reference acceptance. The capture performance is shown for a cooling channel extending to $s=$ $270 \mathrm{~m}$ although in this design the cooling channel extends only to $230 \mathrm{~m}$. Acceptance is maximal at $0.20 \mu$ per initial $8 \mathrm{GeV}$ proton at $s=240 \mathrm{~m}$ ( $85 \mathrm{~m}$ of cooling) and the rms transverse emittance is $7 \mathrm{~mm}$. At $s=230 \mathrm{~m}$ ( $75 \mathrm{~m}$ of cooling) the number of $\mu$ s per proton is 0.19 and the transverse emittance is $7.5 \mathrm{~mm}$.

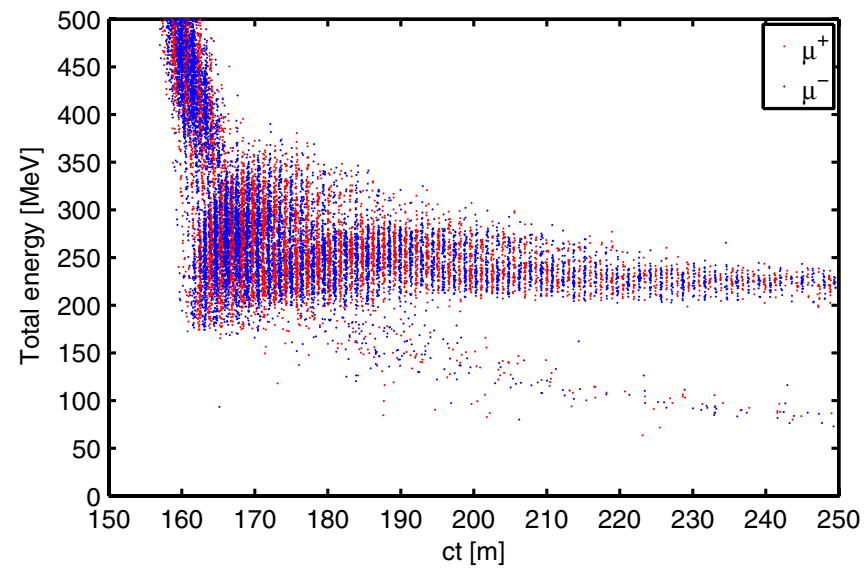

FIG. 5. Distribution of particles in longitudinal phase space at the phase rotation end. $\mu+$ are shown in red and $\mu-$ are shown in blue.

Within the rotator, as the reference particles are accelerated to the central energy (at $p=233 \mathrm{MeV} / c$ ) at the end of the channel, the beam bunches formed before and after the central bunch are decelerated and accelerated respectively, obtaining at the end of the rotator a string of bunches of equal energy for both muon species. At the end of the rotator the $\mathrm{rf}$ frequency matches into the rf frequency of the ionization cooling channel $(201.25 \mathrm{MHz})$. The average momentum at the rotator is $230 \mathrm{MeV} / c$. The performance of the bunching and phase rotation channel, along with the subsequent cooling channel, is displayed in Fig. 4, which shows, as a function of the distance down the channel, the number of muons within a reference acceptance. The phase rotation increases the "accepted" muons by a factor of 4 .

A critical feature of the muon production, collection, bunching, and phase rotation system is that it produces bunches of both signs $\left(\mu^{+}\right.$and $\left.\mu^{-}\right)$at roughly equal intensities. This occurs because the focusing systems are solenoids which focus both signs, and the rf systems have stable acceleration for both signs, separated by a phase difference of $\pi$. The distribution of muons in longitudinal phase space for particles of both signs at the end of the rotator is shown in Fig. 5.

\section{IONIZATION COOLING CHANNEL}

The baseline cooling channel described in [2] remains relatively unchanged. This cooling channel shows a good performance at significant, but probably minimal, cost. The absorber length has been increased by $10 \%$ to take into account modifications to the energy loss in lithium hydride in the two main tracking codes used in this study, ICOOL [7] and G4BEAMLINE [6]. It is hoped that these model uncertainties will be resolved by the muon ionization cooling experiment (MICE) [8] when it operates in 2014.

The main focus of design effort has been on assessing and mitigating the risk associated with the possibility that the rf cavities specified for the front end and especially the cooling channel may break down in strong magnetic fields 
reducing the achievable peak field. Development activity has focused on the cooling channel. While there is a risk of rf breakdown in the buncher and phase rotator, the rf fields and solenoidal fields are lower in here. Additionally, there is no requirement for tight focusing here, unlike in the cooling section, and so it is thought that design of an alternate lattice will be easier.

\section{A. Effect of reduced rf gradient}

The presence of magnetic fields overlapping rf cavities has been identified as a technical risk that may reduce the capture efficiency of the muon front end due to a reduction in the peak gradient that can be achieved in the rf cavities $[9,10]$. Magnetic insulation of rf cavities $[11,12]$ and cooling channels filled with insulating high pressure gas have been proposed as a mode to prevent this breakdown [13]. In Europe the focus has been on shielding cavities from rf fields, both by increasing the cell length of the cooling cell [14] and by reducing magnetic fields in the rf cavities using bucking coils $[15,16]$.

If the rf cavities in the cooling channel fail to reach the desired gradient it can lead to a degradation in muon capture performance. The good muon yield is considered to be the number of muons in a $200 \mathrm{MeV} / c$ momentum bite centered around the reference momentum within a $30 \mathrm{~mm}$ transverse amplitude and a $150 \mathrm{~mm}$ longitudinal acceptance. This is considered to be the nominal acceptance of the downstream acceleration system.

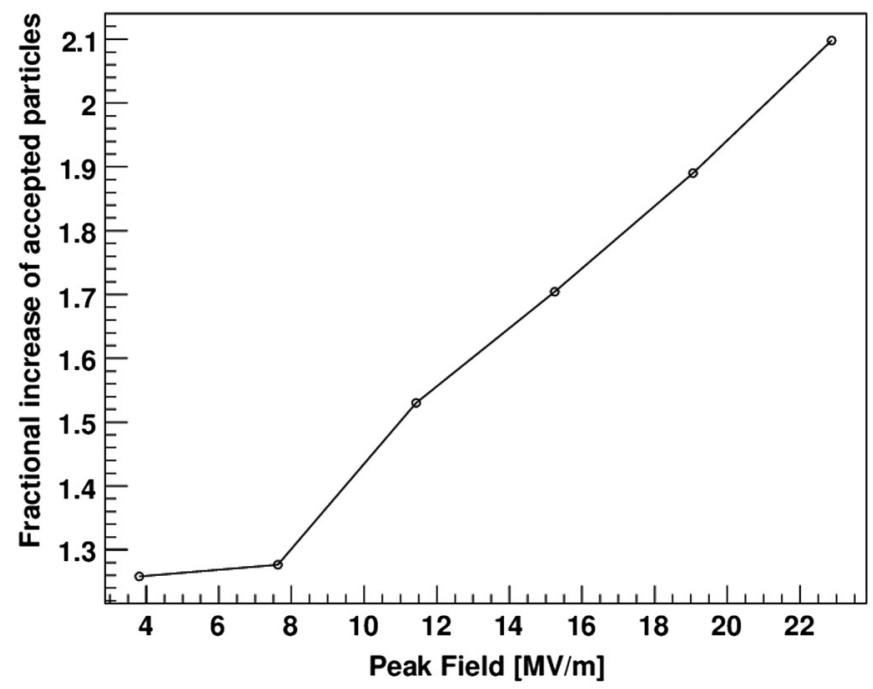

FIG. 6. The degradation in cooling performance with decreased rf voltage is shown. The baseline cooling lattice was simulated with lithium hydride thickness proportional to rf peak voltage to account for the reduction in energy gain and the number of muons within the accelerator acceptance was calculated before and after the cooling channel. The ratio of these two values was plotted against cooling channel rf field for a number of peak fields. The nominal cooling channel rf voltage is $16 \mathrm{MV} / \mathrm{m}$.
The degradation in performance of the baseline cooling channel with rf voltage in the cavities is shown in Fig. 6. The reduction in cooling performance is linear with $\mathrm{rf}$ gradient.

\section{B. Bucked coils}

The principle problem in the ionization cooling channel is to get a tight focus on the emittance absorbers while maintaining a high transverse acceptance. One way to achieve a high acceptance is by tightly packing solenoids, but this leads to overlapping magnetic fields with rf cavities which causes breakdown. The idea of shielding the cavities has been investigated, and yielded some success, but shielding tends to reduce the dynamic aperture of the coils.

The performance of two bucked coil lattices is shown in Fig. 7. Two lattices are shown, one with shielding provided by bucked coils arranged radially and one with shielding provided by bucked coils arranged laterally. The cavity remains in a field around $1 \mathrm{~T}$, and the good muon yield
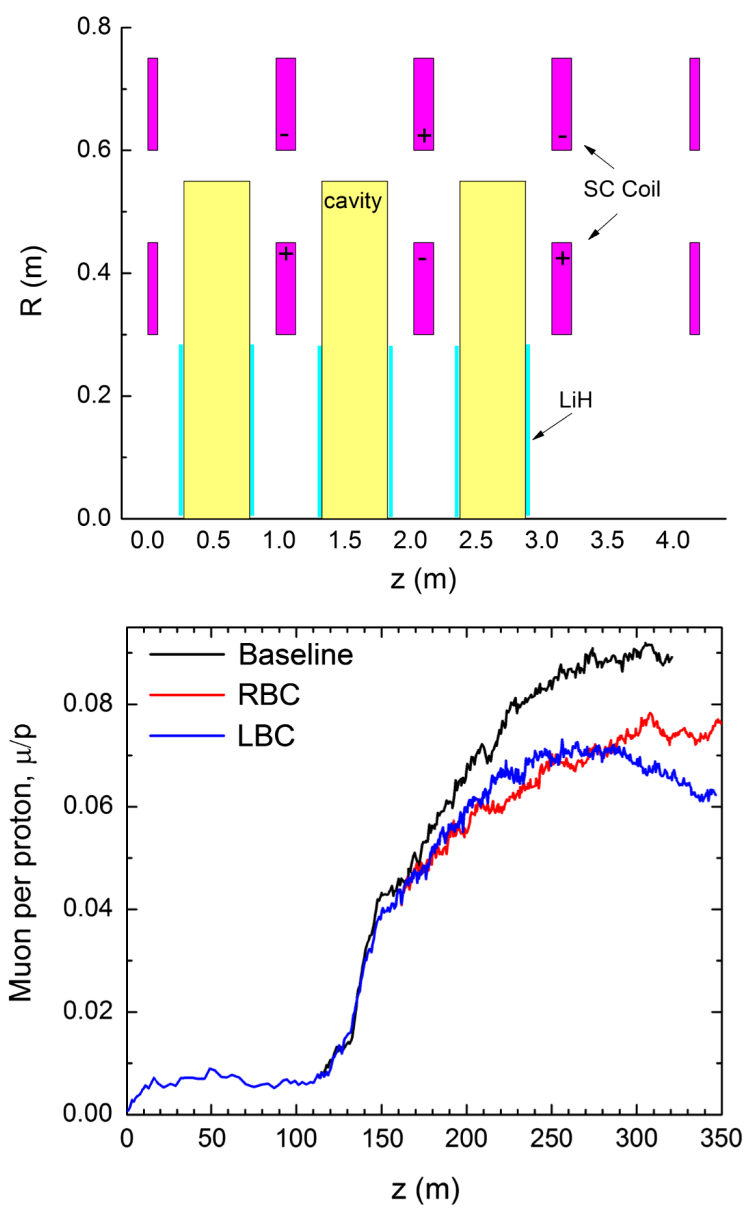

FIG. 7. Schematic of a bucked coil lattice in radially bucked coil (RBC) arrangement and simulated good muon yield with radially bucked coil and laterally bucked coil (LBC) lattices. The bucked coils were used also in the phase rotation section in these simulations. 
is reduced by around $15 \%$. The magnetic field on the cavity is substantially lower, although further experimental guidance is required to confirm that higher gradients can be achieved in such a field.

\section{Increased cell length}

Another way to achieve improved acceptance is to increase the beam energy, taking advantage of the geometrical emittance effect. The rf capture scheme outlined above can capture muons at higher energy by working with a small accelerating phase in the phase rotation section. Operation at higher energy can enable use of a lattice with a reasonable acceptance even with a rather long cell length.

The performance of two lattices with a cell length of $3 \mathrm{~m}$ is shown in Fig. 8. In the first case, a liquid hydrogen absorber is used and the cavity sits in a magnetic field of at worst $1 \mathrm{~T}$. The baseline performance is recovered in this case. In the second case, a lithium hydride absorber is used. As this material is more dense, and safety windows are not required, the absorber and rf assembly can be made shorter so in this case the cavities sit in at worst a $0.3 \mathrm{~T}$ field. The liquid hydrogen absorber is lower $Z$ and so gives better cooling performance recovering the baseline cooling performance, while the use of lithium hydride leads to a worse cooling performance but at lower risk.

The main issue with these lattices is that cooling goes with the fractional decrease in energy. Operation at higher energy requires a larger decrease in energy to get the same emittance reduction, and hence a larger increase in energy, i.e., more rf cavities. The ionization cooling channel comprises a rather large fraction of the total accelerator facility

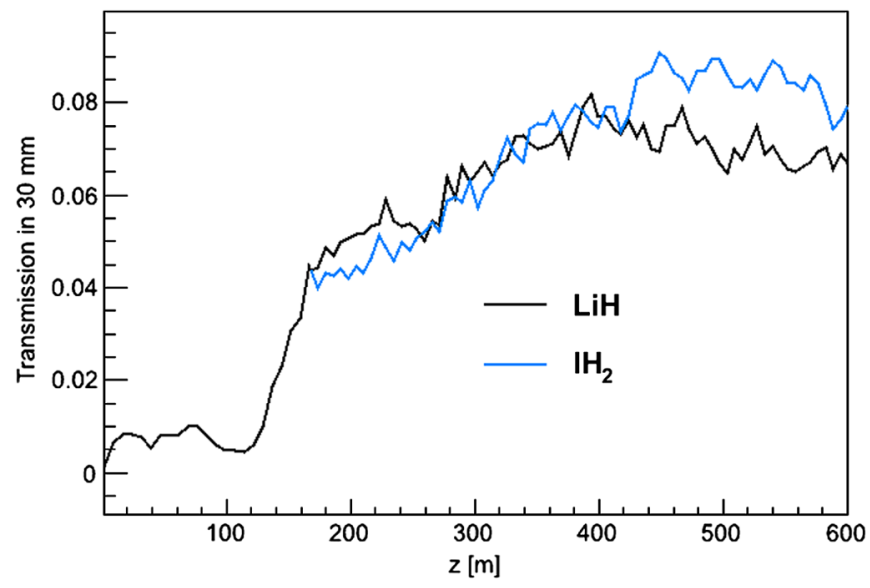

FIG. 8. Simulated good muon yield of $\mu+$ with capture optimized for higher energy and a cooling cell length of $3 \mathrm{~m}$ as opposed to $0.75 \mathrm{~m}$ in the baseline. The rf capture scheme was adjusted to capture at a central momentum of $273 \mathrm{MeV} / c$ rather than the nominal $214 \mathrm{MeV} / c$ of the baseline lattice. Two absorber materials were simulated, lithium hydride $(\mathrm{LiH})$ and liquid hydrogen $\left(\mathrm{lH}_{2}\right)$. cost and the rf cavities comprise the majority of this expense.

\section{SUMMARY}

The neutrino factory muon front end captures a substantial proportion of the muons produced by the neutrino factory target. Longitudinal capture is achieved using a buncher and energy-time phase rotation system while transverse capture is achieved using a high field solenoid adiabatically tapered to $1.5 \mathrm{~T}$ and enhanced by ionization cooling.

Technical risks to the muon front end are presented by the requirement for high peak rf fields in the presence of intense magnetic fields and irradiation of the accelerator hardware due to uncontrolled particle losses. Strategies have been outlined by which these risks can be mitigated. Overall, the muon front end increases the capture rate of muons in the nominal accelerator acceptance by a factor 10 .

\section{ACKNOWLEDGMENTS}

We acknowledge the financial support of the European Community under the European Commission Framework Programme 7 Design Study: EUROnu, Project No. 212372. We also thank colleagues from the International Design Study (IDS-NF) collaboration for fruitful discussions concerning this work.

[1] S. Choubey et al., Rutherford Appleton Laboratory Technical Report No. RAL-TR-2011-018, 2011.

[2] C. Albright et al., The Neutrino Factory and Beta Beam Experiments and Development, available from BNL72369-2004, FNAL-TM-2259, or LBNL-55478, 2004

[3] M. Apollonio et al., JINST, 4, P07001 (2009).

[4] N. V. Mokhov, Fermi National Laboratory Technical Report No. Fermilab-Conf-10-518-APC, 2010.

[5] C. Tunnell and C.T. Rogers, in Proceedings of IPAC2011, San Sebastián, Spain (EPS-AG, Spain, 2011).

[6] T. J. Roberts et al., in Proceedings of EPAC08, Genoa, Italy (EPAC, European Physical Society Accelerator Group, Genoa, Italy, 2008).

[7] R. C. Fernow, in Proceedings of 2005 Particle Accelerator Conference, Knoxville, Tennessee (IEEE, Piscataway, NJ, 2005), pp. 2651-2653.

[8] A. Blondel, in Proceedings of IPAC'12, New Orleans, USA (IEEE, Piscataway, NJ, 2012).

[9] A. Moretti, Z. Qian, J. Norem, Y. Torun, D. Li, and M. Zisman, Phys. Rev. ST Accel. Beams 8, 072001 (2005).

[10] R. B. Palmer, R. Fernow, J. Gallardo, D. Stratakis, and D. Li, Phys. Rev. ST Accel. Beams 12, 031002 (2009).

[11] J. C. Gallardo, D. Stratakis, and R. B. Palmer, J. Phys. G 37, 105011 (2010). 
[12] D. Stratakis, R. C. Fernow, J. C. Gallardo, R. B. Palmer, and D. V. Neuffer, Phys. Rev. ST Accel. Beams 14, 011001 (2011).

[13] J.C. Gallardo and M.S. Zisman, in Proceedings of IPAC'10, Kyoto, Japan (IPAC'10 and Asian Committee for Future Accelerators, Kyoto, 2010), pp. 3515-3517.

[14] C. T. Rogers, in Neutrino Factories, Superbeams and Beta Beams: 11th International Workshop on Neutrino
Factories, Superbeams and Beta Beams-NuFact09, edited by Maury C. Goodman, Daniel M. Kaplan, and Zach Sullivan, AIP Conf. Proc. No. 1222 (American Institute of Physics, Melville, NY, 2010), pp. 298-302.

[15] A. Alekou, Ph.D. thesis, Imperial College, London, 2012.

[16] A. Alekou and J. Pasternak, JINST 7, 08017 (2012). 